\title{
Hydrogen sulfide in the RVLM and PVN has no effect on cardiovascular regulation
}

\author{
Eloise Streeter ${ }^{1,2}$, Mohammad Al-Magableh ${ }^{1,2}$, Joanne Louise Hart ${ }^{1,2}$ and Emilio Badoer ${ }^{1,2}$ * \\ School of Medical Sciences, RMIT University, Melbourne, VIC, Australia \\ ${ }^{2}$ Health Innovations Research Institute, RMIT University, Melbourne, VIC, Australia
}

Edited by:

Elisabeth Lambert, Baker IDI Heart and Diabetes Institute, Australia

Reviewed by:

Roger Evans, Monash University, Australia

Dmitry Mayorov, University of

Melbourne, Australia

${ }^{*}$ Correspondence:

Emilio Badoer, School of Medical Sciences, RMIT University, PO Box 71, Bundoora 3083, VIC, Australia. e-mail:emilio.badoer@rmit.edu.au
Hydrogen sulfide $\left(\mathrm{H}_{2} \mathrm{~S}\right)$ is now recognized as an important signaling molecule and has been shown to have vasodilator and cardio-protectant effects. More recently it has been suggested that $\mathrm{H}_{2} \mathrm{~S}$ may also act within the brain to reduce blood pressure (BP). In the present study we have demonstrated the presence of the $\mathrm{H}_{2} \mathrm{~S}$-producing enzyme, cystathionine- $\beta$-synthase (CBS) in the rostral ventrolateral medulla (RVLM), and the hypothalamic paraventricular nucleus (PVN), brain regions with key cardiovascular regulatory functions. The cardiovascular role of $\mathrm{H}_{2} \mathrm{~S}$ was investigated by determining the $\mathrm{BP}$, heart rate (HR), and lumbar sympathetic nerve activity (LSNA) responses elicited by a $\mathrm{H}_{2} \mathrm{~S}$ donor sodium hydrogen sulfide (NaHS) or inhibitors of CBS, microinjected into the RVLM and PVN. In anesthetized Wistar Kyoto rats bilateral microinjections of NaHS $(0.2-2000 \mathrm{pmol} / \mathrm{side})$ into the RVLM did not significantly affect BP, HR, or LSNA, compared to vehicle. Similarly, when the CBS inhibitors, amino-oxyacetate $(A O A ; 0.1-1.0 \mathrm{nmol} /$ side) or hydroxylamine $(\mathrm{HA}$; $0.2-2.0 \mathrm{nmol} / \mathrm{side})$, were administered into the RVLM, there were no significant effects on the cardiovascular variables compared to vehicle. Microinjections into the PVN of NaHS, $H A$, and $A O A$ had no consistent significant effects on BP, HR, or LSNA compared to vehicle. We also investigated the cardiovascular responses to NaHS microinjected into the RVLM and PVN in spontaneously hypertensive rats. Again, there were no significant effects on $\mathrm{BP}, \mathrm{HR}$, and LSNA. Together, these results suggest that $\mathrm{H}_{2} \mathrm{~S}$ in the RVLM and PVN does not have a major role in cardiovascular regulation.

Keywords: hydrogen sulfide, paraventricular nucleus, rostral ventrolateral medulla, lumbar sympathetic nerve activity, cardiovascular

\section{INTRODUCTION}

Hydrogen sulfide $\left(\mathrm{H}_{2} \mathrm{~S}\right)$ is better known for its pungent odor and toxic properties than its potential therapeutic actions. It is becoming increasingly clear, however, that $\mathrm{H}_{2} \mathrm{~S}$, like other gaseous transmitters, such as nitric oxide (NO), is an important endogenous signaling molecule in many physiological functions (Abe and Kimura, 1996; Ufnal et al., 2008; Yang et al., 2008; Calvert et al., 2009; Hart, 2011). In mammals $\mathrm{H}_{2} \mathrm{~S}$ is produced endogenously in the body from the catabolism of amino acids, such as cysteine and homocysteine predominantly by the pyridoxal $-5^{\prime}$-phosphatedependant enzymes - Cystathionine- $\gamma$-lyase (CSE, EC 4.4.1.1), and Cystathionine- $\beta$-synthase (CBS, EC 4.2.1.22; Kabil and Banerjee, 2010). CSE is found primarily in the periphery and CBS is a major enzyme responsible for the production of $\mathrm{H}_{2} \mathrm{~S}$ in the central nervous system (Lee et al., 2009; Mustafa et al., 2009).

Hydrogen sulfide has a number of effects including neuromodulation, anti-oxidant, anti-inflammatory, and cardiovascular actions (Kimura, 2002; Mustafa et al., 2009; Gadalla and Snyder, 2010). In the cardiovascular system, $\mathrm{H}_{2} \mathrm{~S}$ has been reported to have positive properties including protective effects against cardiac ischemia-reperfusion injury (Calvert et al., 2009) and peripheral vasodilatory effects. $\mathrm{H}_{2} \mathrm{~S}$ donors, such as $\mathrm{NaHS}$, have been shown to relax blood vessels in vitro (Zhao and Wang, 2002; Cheang et al., 2010; Al-Magableh and Hart, 2011) and systemic administration of NaHS reduces blood pressure (BP) in a dose-dependent manner (Zhao et al., 2001). Recent work using CSE knockout mice has suggested that endogenously produced $\mathrm{H}_{2} \mathrm{~S}$ is necessary for maintaining normal BP since these animals develop hypertension and have an attenuated endothelium-dependent vasorelaxation (Yang et al., 2008).

Hydrogen sulfide is also produced in the central nervous system (Kimura, 2002; Mustafa et al., 2009; Gadalla and Snyder, 2010), as is the case for the gaseous transmitter, nitric oxide. Recently, it has been reported that $\mathrm{H}_{2} \mathrm{~S}$ administered into the lateral brain ventricles of conscious rats resulted in small but significant increases in BP (Ufnal et al., 2008). That finding suggested that $\mathrm{H}_{2} \mathrm{~S}$ could influence cardiovascular regulation via actions within the central nervous system. To gain an insight into the specific nuclei within the brain in which $\mathrm{H}_{2} \mathrm{~S}$ is acting, a recent study investigated the role of the posterior hypothalamus in conscious rats (Dawe et al., 2008). In that study a small reduction in BP was observed, the opposite to that observed following lateral ventricular administration of $\mathrm{H}_{2} \mathrm{~S}$. Taken together, the evidence to date suggests that $\mathrm{H}_{2} \mathrm{~S}$ can act in the brain to influence BP, but that the posterior hypothalamus is not the only nucleus involved.

Regulating the activity of the sympathetic nervous system is a key mechanism through which the brain can influence the level of BP. There are several areas in the brain that are known 
to directly influence sympathetic nerve activity (SNA) via direct projections to the intermediolateral cell column of the spinal cord, where sympathetic preganglionic motor neurons are located. These key autonomic regions have important cardiovascular regulatory functions and include the rostral ventrolateral medulla (RVLM) and the paraventricular nucleus (PVN) of the hypothalamus (Shafton et al., 1998; Guyenet, 2006). The RVLM plays a pivotal role in the tonic and reflex control of sympathetic vasomotor activity, such that bilateral inhibition or destruction of neurons in the RVLM results in dramatic decreases in both arterial pressure and sympathetic vasomotor activity (Guyenet, 2006). The $\mathrm{PVN}$ is a major integrative nucleus that can markedly influence BP, SNA, and the hemodynamic sequelae (Badoer, 2001, 2010). Activation of the PVN can elicit increases or decreases in SNA and blood flow (Deering and Coote, 2000; Badoer, 2001), suggesting both sympatho-inhibitory and sympatho-excitatory outflows may emanate from the PVN.

Very recently, a report appeared in which the effects of microinjecting NaHS into the RVLM on renal SNA was investigated. Whether NaHS microinjected into the RVLM can influence SNA to other vascular organs and whether it can act in the PVN to influence SNA is unclear. Additionally, there is no data available on whether $\mathrm{H}_{2} \mathrm{~S}$ acting in those brain regions has different effects in normotensive and hypertensive conditions. Therefore, the aim of the present study was to determine whether $\mathrm{H}_{2} \mathrm{~S}$ could alter lumbar sympathetic nerve activity (LSNA), BP, and heart rate (HR) by acting within the RVLM or PVN in normotensive as well as hypertensive rats.

\section{MATERIALS AND METHODS ANIMALS}

Male wistar kyoto (WKY) and spontaneously hypertensive (SHR) rats, weighing 300-350 g, were obtained from the Animal Resources Centre (ARC, Canning Vale, WA, Australia). The animals were housed in a temperature-controlled room on a $12: 12 \mathrm{~h}$ light/dark cycle (lights on at 7:00 a.m.), in the RMIT Animal Facility (RMIT University, Bundoora West campus, VIC, Australia). The animals were housed for a minimum period of 1 week before undergoing any experimental procedure. All procedures were performed to conform to the guidelines set out by the National Health and Medical Research Council of Australia and were approved by the RMIT University Animal Ethics committee.

\section{SURGICAL PROCEDURES}

Rats were anesthetized initially with inhaled isoflurane (1-3\% in air), by placing the animal into a sealed container which was subsequently filled with the gas. Once anesthesia was induced, isoflurane was continually administered via a mask while the femoral vein and artery were cannulated as described previously (Chen et al., 2008). Briefly, the right femoral vein and artery were exposed by blunt dissection and vein and artery were cannulated separately using two polyethylene catheters consisting of PE 10 tubing connected to PE 50 tubing, filled with heparinized saline $(50 \mathrm{U} / \mathrm{ml})$. Anesthesia was then maintained using urethane (1-1.5 g/kg iv) with supplemental doses as required $(0.1-0.3 \mathrm{~g} / \mathrm{kg}$ iv), administered through the cannulated vein. The depth of anesthesia was maintained to ensure the absence of corneal and pedal reflexes. The distal end of the arterial cannula was attached to a BP transducer for direct monitoring of BP.

Following a midline abdominal incision, the left lumbar postganglionic sympathetic nerve trunk was identified and dissected free of surrounding tissue. With the aid of an operating microscope the nerve was placed onto the bared tips of two Teflon-coated silver wire electrodes and the nerve-electrode junction insulated electrically from surrounding tissue with a sealant (Kwik-Cast Sealant, WPI, USA). The nerve activity was amplified using a low-noise differential amplifier (ENG Models 187B and 133, Baker Institute, VIC, Australia), filtered (bandpass $100-1000 \mathrm{~Hz}$ ), rectified, and integrated at $0.5 \mathrm{~s}$ intervals. The signal was recorded using a MacLab data acquisition system (ADInstruments, NSW, Australia). The signal recorded at the end of the experiment after the injection of phenylephrine $(5 \mu \mathrm{g} / \mathrm{kg}$, iv) was deemed background noise. The LSNA was calculated by subtraction of background noise from the recorded nerve activity. The average integrated LSNA was calculated over a period of 1-2 min and expressed as a percentage of the resting period prior to the intracerebral administration of drugs.

\section{Microinjections into the RVLM and Hypothalamus}

For microinjections into the RVLM, each animal was placed prone and the head was mounted in a Stoelting stereotaxic frame such that both bregma and lambda were positioned on the same horizontal plane. Burr holes were drilled bilaterally into the occipital bone of the skull approximately $2 \mathrm{~mm}$ lateral of the mid-sagittal suture and $3.8 \mathrm{~mm}$ caudal of the lambdoid suture. The pressor region of the RVLM was identified functionally by microinjection of $50 \mathrm{nl}$ of L-glutamate $(0.1 \mathrm{M})$ which elicited a pressor response of at least $20 \mathrm{mmHg}$ in arterial pressure (Kantzides et al., 2005). RVLM microinjections were made using the following coordinates; $3.7-4.0 \mathrm{~mm}$ caudal to lambdoid suture, $2 \mathrm{~mm}$ lateral to the midline, and $8.0 \mathrm{~mm}$ ventral to the surface of the dura.

For microinjections into the hypothalamic PVN, a midline reference point was marked $2 \mathrm{~mm}$ rostral to bregma. This was necessary because bregma was removed in some instances during the subsequent bone drilling procedure. Holes (approximately $4 \mathrm{~mm}$ in diameter) were drilled bilaterally into the skull centered $4.0 \mathrm{~mm}$ caudal from the reference point to allow microinjections of drugs into the PVN (stereotaxic coordinates: $3.8-4.1 \mathrm{~mm}$ caudal to the reference point, $0.5 \mathrm{~mm}$ lateral to midline, and $8.0 \mathrm{~mm}$ ventral to the surface of the dura).

All microinjections were made bilaterally using a fine glass micropipette (with a tip diameter of $50-70 \mu \mathrm{m}$ ). Microinjection volumes were $100 \mathrm{nl} /$ side and after each microinjection, the micropipette was left in place for approximately $1 \mathrm{~min}$. To mark the injection sites, a small amount of rhodamine-tagged fluorescent microspheres was included in the microinjected solution (LumaFluor, NC, USA). The precise location of the microinjections was verified histologically at the end of each experiment.

\section{EXPERIMENTAL PROTOCOL}

In WKY rats bilateral microinjections were made into the RVLM $(n=16), \operatorname{PVN}(n=18)$, and into the area adjacent to the PVN $(n=8)$. Animals receiving microinjections into the RVLM were given vehicle (artificial CSF containing $\mathrm{NaCl} 124 \mathrm{mM}$, 
$\mathrm{KCl} 3.0 \mathrm{mM}, \mathrm{NaH}_{2} \mathrm{PO}_{4} \cdot 2 \mathrm{H}_{2} \mathrm{O} 1.3 \mathrm{mM}, \mathrm{MgCl}_{2} \cdot 6 \mathrm{H}_{2} \mathrm{O} 2.0 \mathrm{mM}$, $\mathrm{NaHCO}_{3} 26 \mathrm{mM}$, glucose $10 \mathrm{mM}, \mathrm{CaCl}_{2} 2.0 \mathrm{mM}$ in Milli-Q water, buffered with carbogen), followed by either (i) five sequential doses of NaHS $(0.2,2,20,200$, and $2000 \mathrm{pmol} / \mathrm{side})$ or (ii) hydroxylamine (HA; 0.2 , and $2 \mathrm{nmol} /$ side, sequentially), and amino-oxyacetate (AOA; 0.1 and $1 \mathrm{nmol} /$ side, sequentially) the order of $\mathrm{HA}$ and AOA was randomized. For microinjections into or out of the PVN the same protocol was followed except only three sequential doses of $\mathrm{NaHS}$ were administered $(20,200$, and $2000 \mathrm{pmol} / \mathrm{side})$. In three additional anesthetized rats, a bolus dose of NaHS (20 nmol) was administered into the lateral cerebral ventricle to determine the effects on the cardiovascular variables. In SHR rats, vehicle and NaHS (20-2000 pmol/side) were microinjected into the RVLM $(n=3)$ and PVN $(n=4)$ following a similar protocol. For all experiments, 10-15 min were allowed between each microinjection of drug. Mean arterial pressure (MAP), HR, and LSNA were monitored continuously. Resting levels prior to drug administration were recorded at $20 \mathrm{~min}$ before and immediately prior to the first intracerebral microinjection. At 1, 5, and $10 \mathrm{~min}$ after the administration of each dose of drug, MAP, HR, and LSNA were recorded for a duration of 1-2 min.

\section{HISTOLOGY}

At the end of each experiment, rats were killed using an overdose of pentobarbital sodium (325 mg/kg; Lethabarb, Virbac, NSW, Australia). The brain of each rat was then carefully removed and placed in a solution of $4 \%$ paraformaldehyde and $20 \%$ sucrose for 1 week. The medulla (for brains which had been microinjected into the RVLM) or the hypothalamus (for brains which had been microinjected into the PVN) were cut on a cryostat into $40 \mu \mathrm{m}$-thick sections and mounted onto gelatine subbed slides. The sections were then viewed wet under fluorescent microscopy to determine the position of the rhodamine beads which indicated the microinjection site. For the medulla, the caudal end of the facial nucleus, the nucleus ambiguous and the inferior olivary nuclei were identified in the wet sections, and the microinjection sites were mapped in relation to those structures. For the hypothalamus, after the center of the microinjections site was identified, the sections were dried before being stained with cresyl violet and cover-slipped with Depex mounting medium (BDH Lab Supplies, Poole, UK). Light microscopy was then used to re-examine the stained hypothalamic sections to determine the extent of the PVN and adjacent anatomical structures. The microinjection sites were subsequently mapped in relation to the PVN and the anatomical structures.

\section{DETECTION OF $\mathrm{H}_{2}$ S-PRODUCING ENZYMES VIA SDS-PAGE AND WESTERN BLOTTING}

Wistar kyoto rat brains were used for western blot analysis of CSE and CBS. The RVLM $(n=3)$ or hypothalamic PVN $(n=3)$ were punched out from frozen sections encompassing the entire rostral-caudal extent of each nucleus, using a blunted 20G needle. For the PVN the tissues from three animals were combined, as were those from the RVLM. The tissues were homogenized and suspended in sample buffer (sample buffer composition: 5\% v/v Glycerine, $2.5 \% \mathrm{v} / \mathrm{v}$ mercaptoethanol, $1.5 \%$ SDS, $0.05 \mathrm{M} \mathrm{TRIS} / \mathrm{HCl}$ $\mathrm{pH} 8,0.05 \mathrm{mg} / \mathrm{ml}$ bromophenol blue). Samples were then heated to $65^{\circ} \mathrm{C}$ for $10 \mathrm{~min}$. Protein concentration was determined from each sample and the samples were loaded onto $10 \%$ gels and separated by SDS-PAGE. After transfer to polyvinylidene difluoride membranes the blots were incubated with primary antibodies suspended in blocking buffer overnight [rabbit anti-CSE antibody (Proteintech Group Inc., USA) and mouse anti-CBS antibody (Abnova Corporation, Taiwan)]. The blots were then incubated with the appropriate secondary antibody (goat anti-rabbit, goat anti-mouse) conjugated to horseradish peroxidase for $1 \mathrm{~h}$ then developed by enhanced chemiluminescence (Millipore Kit). Dual color marker (Bio-Rad) was used for molecular weight determination. Recombinant protein of CSE and CBS (GST-tagged) were loaded on the gel to identify the band of interest (Abnova, Taiwan).

\section{STATISTICS}

The data from the in vivo studies were expressed as the change between the level immediately prior to each microinjection and the average of the level observed at 1 and $5 \mathrm{~min}$ after drug/vehicle administration. These time points corresponded to those used by others (Dawe et al., 2008) and to the times at which an effect was most likely to be observed, as exemplified by the time course of effects following NaHS (data not shown). Since the vehicle was similar in each experiment, the vehicle responses were combined into a single control group for each brain region.

The average value of the changes was calculated and was subsequently compared between groups using one-way-ANOVA, followed by comparisons between the individual doses of drugs and control using Dunnett's post hoc test for multiple comparisons. $P<0.05$ (two-tailed) was considered statistically significant.

\section{RESULTS}

\section{WKY RATS}

\section{CBS and CSE in RVLM and PVN}

Figure 1A shows examples of the western blots used to determine the presence of CSE and CBS in the RVLM and PVN in the rat brain. The results show that the PVN and RVLM contain CBS. By contrast, in neither region was CSE detectable. As positive controls, we have previously shown that CSE is found in peripheral tissues such as the aorta and kidney (Al-Magableh and Hart, 2011).

\section{Effect of NaHS microinjected into the RVLM}

Sodium hydrogen sulfide (0.2-2000 pmol/side) microinjected into the RVLM resulted in small increases in MAP and HR but these were not significantly different from vehicle (Figure 1B). The LSNA responses were small and variable; LSNA decreased slightly following NaHS $(0.2-20 \mathrm{pmol})$ and slightly increased or did not change following the higher doses, but was not significantly different from the vehicle response (Figure 1B).

\section{Effect of HA and AOA microinjected into the rostral RVLM}

The CBS inhibitors, AOA (0.1-1.0 nmol/side), and HA (0.2$2.0 \mathrm{nmol} / \mathrm{side}$ ) microinjected into the RVLM did not significantly change any of the cardiovascular variables measured compared to vehicle (Figure 2). Following the highest dose of AOA, MAP, and HR tended to decrease but this was not seen with HA. Neither AOA nor HA significantly affected LSNA. 


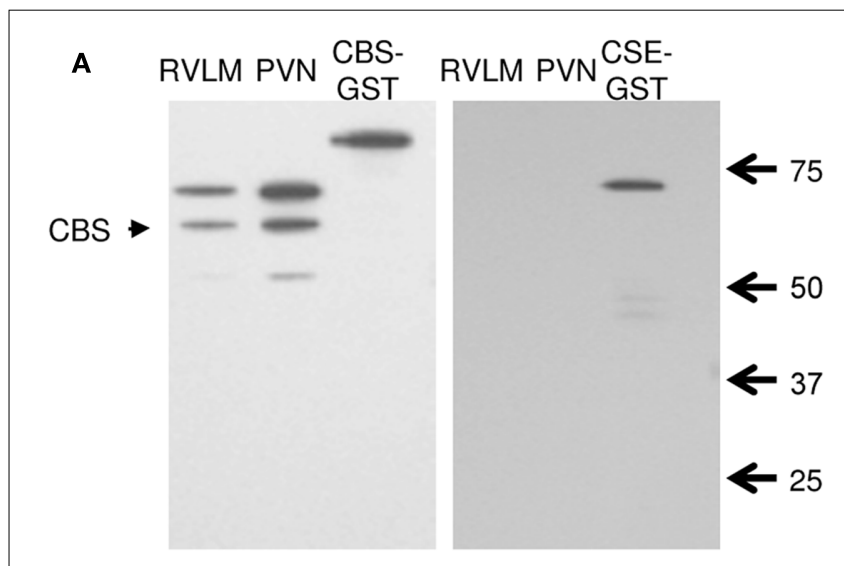

B
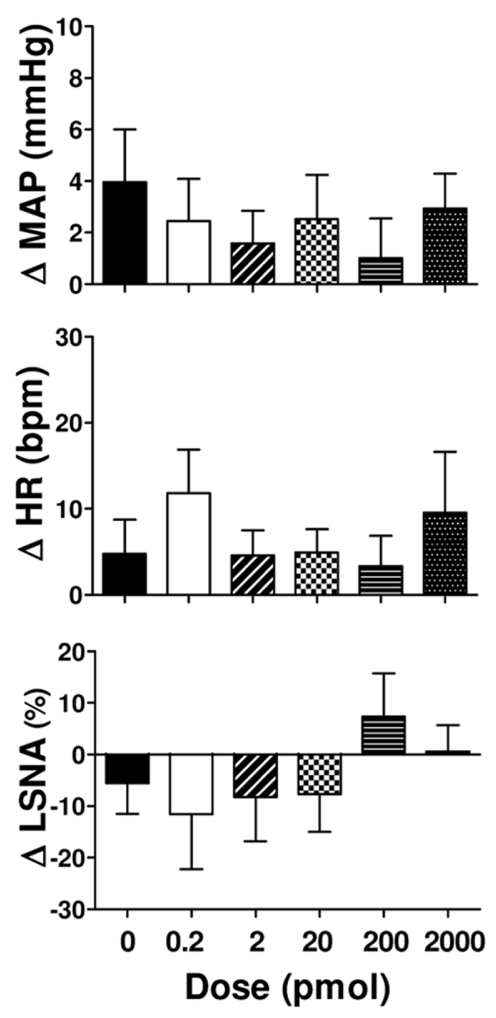

FIGURE 1 | (A) Western blot showing expression of cystathionine- $\beta$-synthase (CBS) and cystathionine- $\gamma$-lyase (CSE) in punched out homogenates of the rostral ventrolateral medulla (RVLM) and hypothalamic paraventricular nucleus (PVN). The $63 \mathrm{kDa}$ band corresponding to CBS protein was labeled in both PVN and RVLM samples. No CSE was observed in the PVN or RVLM. The GST-tagged CBS ( $86 \mathrm{kDa})$ and GST-tagged CSE (70 kDa) are shown. The native CSE protein is $44 \mathrm{kDa}$. (B) Changes in mean arterial pressure (MAP), heart rate (HR), and lumbar sympathetic nerve activity (LSNA) following vehicle ( $n=13$ for MAP and HR and $n=6$ for LSNA) and the $\mathrm{H}_{2} \mathrm{~S}$ donor (NaHS, $0.2-2000 \mathrm{pmol} / \mathrm{side} ; n=8$ for MAP and HR; and $n=5$ for LSNA) microinjected into the rostral ventrolateral medulla of WKY rats. Different shaded bars represent a different dose of $\mathrm{NaHS}$ as indicated.

\section{Effect of NaHS microinjected into the PVN}

Microinjection of NaHS $(20-2000 \mathrm{pmol} / \mathrm{side})$ into the PVN slightly increased MAP but this was not significantly different from the vehicle response (Figure 3). Similarly, the average

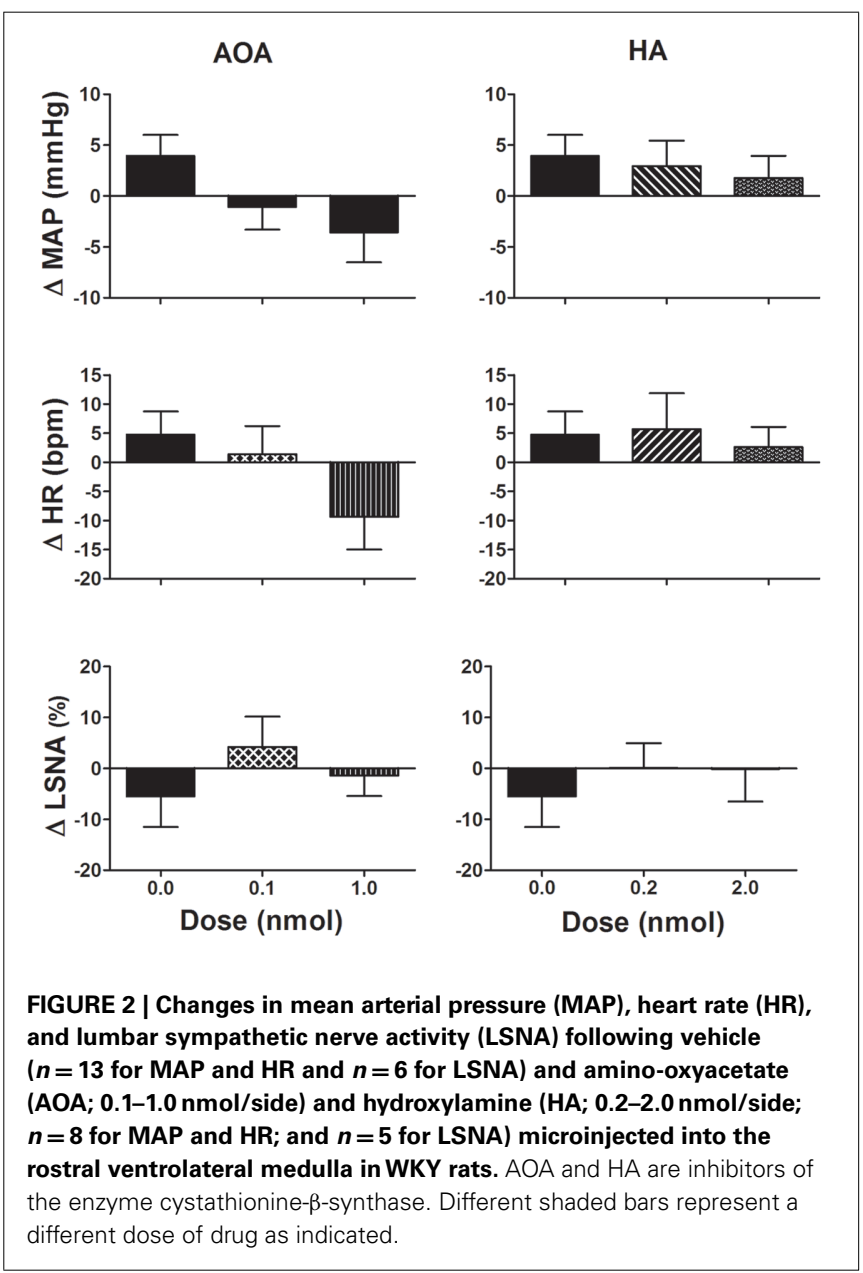

changes in HR following NaHS were not significantly different from the vehicle response (Figure 3). LSNA was not markedly affected by microinjection of NaHS into the PVN (Figure 3). NaHS microinjected into the area surrounding the PVN also had no significant effect on MAP, HR, or LSNA ( $n=4$; data not shown).

\section{Effect of HA and AOA microinjected into the PVN}

Microinjection of AOA (0.1-1.0 nmol/side) into the PVN produced no significant change in MAP, HR, or LSNA compared to vehicle (Figure 4). Microinjection of HA $(0.2 \mathrm{nmol} /$ side $)$ into the PVN resulted in a small but significant decrease in MAP and HR compared to vehicle (Figure 4). Microinjection of the higher dose of HA ( $2.0 \mathrm{nmol} / \mathrm{side})$ into the PVN, however, did not elicit any significant effect on MAP and HR compared to vehicle (Figure 4). Neither dose of HA had any significant effect on LSNA compared to vehicle. Additionally, AOA and HA microinjected into the area surrounding the PVN also had no significant effect on MAP, HR, or LSNA ( $n=4$; data not shown).

\section{Intracerebroventricular administration of $\mathrm{NaHS}$}

When NaHS $(20 \mathrm{nmol})$ was administered into the lateral cerebral ventricle there were no significant effects on MAP (average change $=0.1 \pm 1.6 \mathrm{mmHg}), \mathrm{HR} \quad(-5 \pm 3 \mathrm{~b} / \mathrm{min})$, or LSNA $(0.2 \pm 8.4 \%)$. 

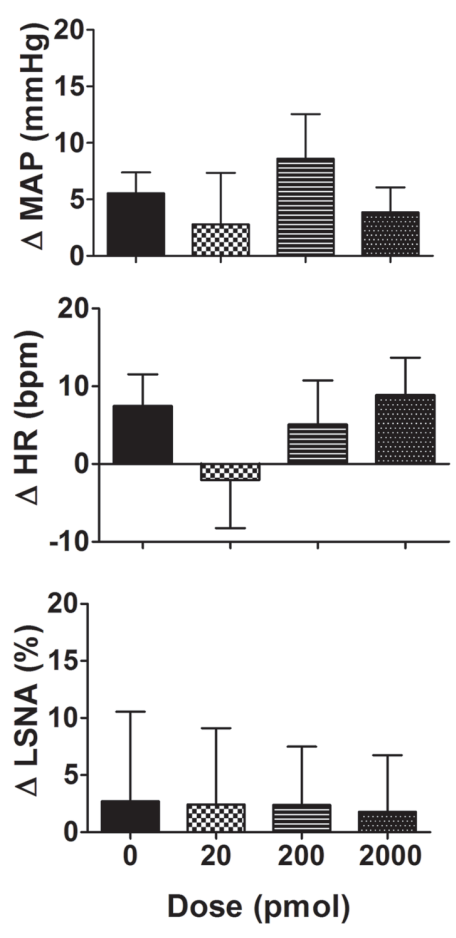

FIGURE 3 | Changes in mean arterial pressure (MAP), heart rate (HR), and lumbar sympathetic nerve activity (LSNA) following vehicle ( $n=14$ for MAP and HR and $n=7$ for LSNA) and the $\mathrm{H}_{2} \mathrm{~S}$ donor, NaHS (20-2000 pmol/side; $n=6$ for MAP and HR and $n=5$ for LSNA) microinjected into the hypothalamic paraventricular nucleus in WKY rats. Different shaded bars represent a different dose of NaHS as indicated.

\section{SHR Rats}

The average resting MAP prior to the microinjections into the brain in the SHR rats was $96.4 \pm 3.9 \mathrm{mmHg}(n=7)$ which was significantly greater than in the WKY rats $(81.3 \pm 2.0, n=34$; $P<0.001)$. HR, however, was not significantly different between SHR and WKY rats $(343 \pm 9$ vs $332 \pm 6, P<0.001)$.

\section{Microinjection into the RVLM}

When NaHS (20-200 pmol/side) was microinjected into the RVLM of SHR rats, there was no significant effect on MAP, and a slight increase following the $2000 \mathrm{pmol}$ dose. In no instance was there any statistically significant difference from vehicle $(n=3$; Figure 5A). On average, a small tachycardia was observed following each dose of NaHS but this was not significantly different from the vehicle response. LSNA tended to increase with the lower doses and decreased with the highest dose of NaHS but there was no significant difference compared to vehicle (Figure 5A).

\section{Microinjection into the PVN}

Microinjections of NaHS into the PVN of SHR rats did not significantly affect MAP, HR, or LSNA ( $n=5$; Figure 5B).

\section{Microinjection sites in the RVLM}

The sites of microinjection into the RVLM (defined as within 0.0 $0.6 \mathrm{~mm}$ caudal of the facial nucleus) are shown in Figure 6A. All microinjection sites were mainly located toward the rostral end of the pressor region of the RVLM predominantly within $0.0-0.2 \mathrm{~mm}$

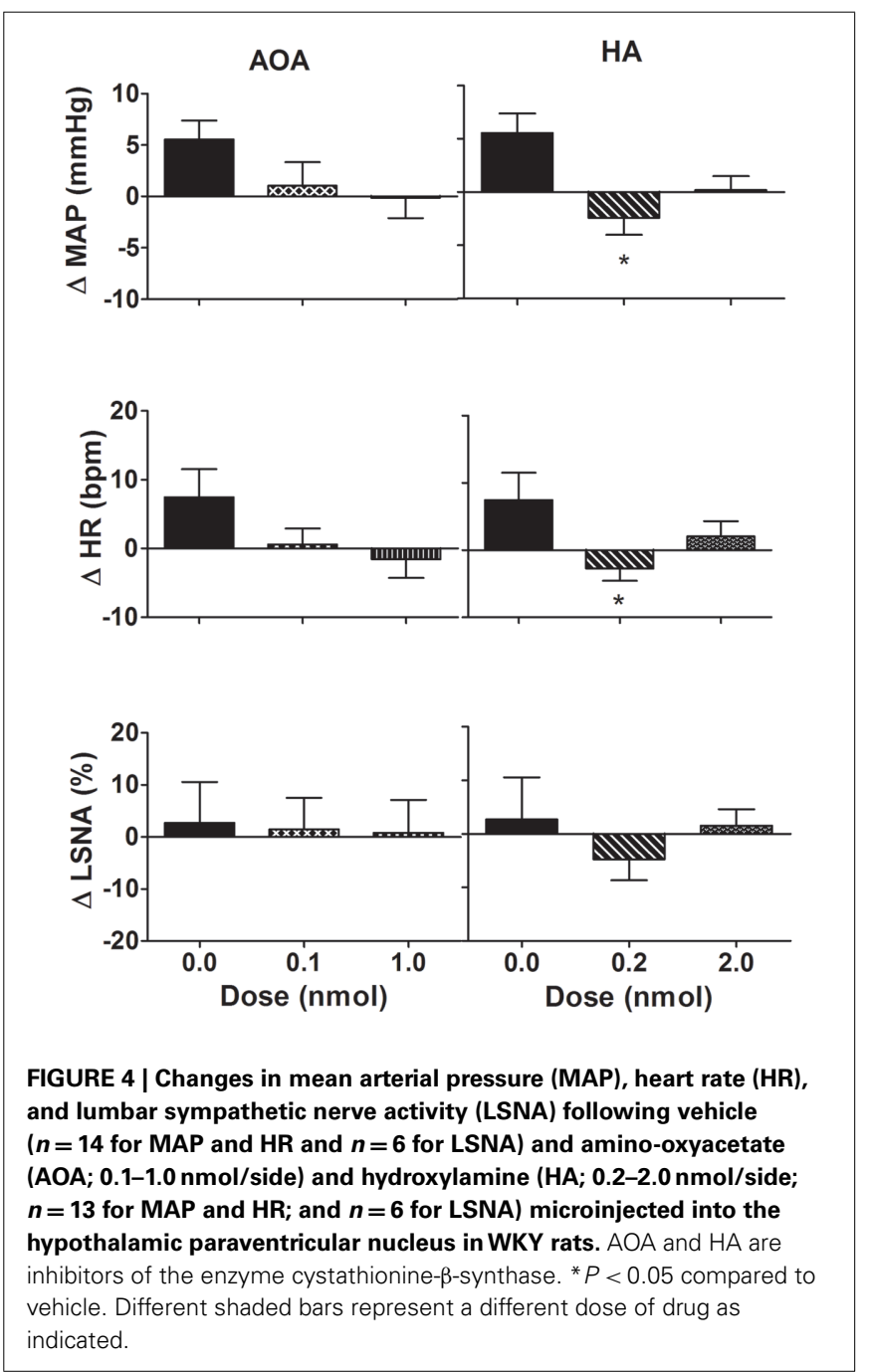

caudal to the caudal pole of the facial nucleus. The distribution of the microinjection sites for NaHS, AOA, and HA were similar.

\section{Microinjection sites in the PVN}

The sites of microinjection into the PVN are shown in Figure 6B. The center of the injection sites were found to be within 1.4-2.1 mm caudal to the bregma and covered the rostral-caudal extent of the PVN. The distribution of the microinjection sites with $\mathrm{NaHS}$ and that of the inhibitors of the enzyme that produces $\mathrm{H}_{2} \mathrm{~S}$, were similar. Microinjections made adjacent to the PVN were centered dorsal to the PVN, in the ventral portion of the nucleus reuniens, at levels $1-2.1 \mathrm{~mm}$ caudal to bregma (data not shown).

\section{DISCUSSION}

In the present study we found that the enzyme CBS but not CSE was present in the pressor region of the RVLM and in the PVN, suggesting $\mathrm{H}_{2} \mathrm{~S}$ may be endogenously produced in these brain regions. Microinjection of the $\mathrm{H}_{2} \mathrm{~S}$ donor, NaHS, directly into these regions, however, did not significantly alter MAP, HR, or LSNA in WKY and SHR rats. In WKY rats, inhibition of the production of $\mathrm{H}_{2} \mathrm{~S}$, using inhibitors of CBS, in those brain regions also had no marked or consistent effects on the cardiovascular 

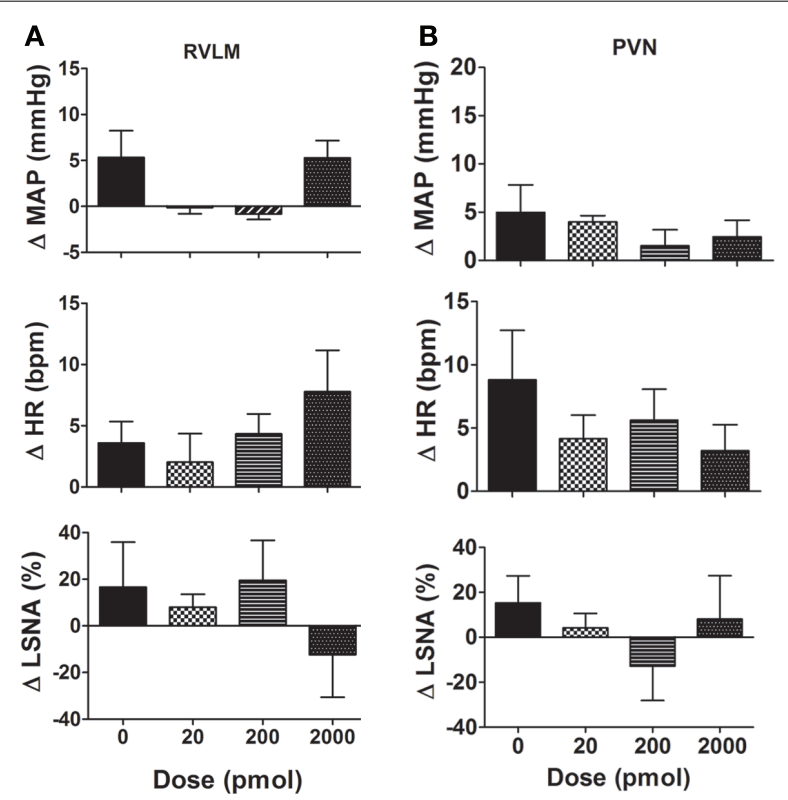

FIGURE 5 | (A) Changes in mean arterial pressure (MAP), heart rate (HR), and lumbar sympathetic nerve activity (LSNA) following vehicle and the $\mathrm{H}_{2} \mathrm{~S}$ donor (NaHS, 20-2000 pmol/side; $n=3$ ) microinjected into the rostral ventrolateral medulla (RVLM) of SHR rats. Different shaded bars represent a different dose of NaHS as indicated. (B) Changes in mean arterial pressure (MAP), heart rate (HR), and lumbar sympathetic nerve activity (LSNA) following vehicle and the $\mathrm{H}_{2} \mathrm{~S}$ donor, NaHS (20-2000 pmol/side; $n=5$ ) microinjected into the hypothalamic paraventricular nucleus (PVN) in SHR rats. Different shaded bars represent a different dose of $\mathrm{NaHS}$ as indicated.

variables. The results suggest that hydrogen sulfide in the RVLM and PVN does not have a significant role in cardiovascular regulation.

The present work, using western blot analysis, is the first to report that CBS is present specifically in the RVLM and PVN, two important autonomic brain regions involved in the regulation of SNA and the cardiovascular system. Previous studies have found that the activity of CBS varies with the brain area examined, and that the hypothalamus had the highest activity (Kohl and Quay, 1979). Changes in CBS expression have also been observed in mice during development; the levels of CBS increased postnatally, particularly in the hippocampus and cerebellum (Robert et al., 2003). There have been reports demonstrating weak expression of CSE in whole brain homogenates (Abe and Kimura, 1996; Ishii et al., 2004), however, our results indicate that CSE is absent in the RVLM and PVN. Taken together, the evidence indicates CBS is the most abundant of the two enzymes in the brain, and that CSE may be present in very limited amounts in brain but not in the specific autonomic regions of the RVLM and the hypothalamic PVN.

The hypothalamic PVN can influence the cardiovascular system via hormonal and neural mechanisms. In the present study, however, NaHS microinjected into the PVN of WKY rats did not significantly influence MAP, HR, or LSNA compared to the microinjection of vehicle. Similarly, neither HA nor AOA into the PVN elicited consistent significant responses. Thus, we conclude that exogenous administration of the $\mathrm{H}_{2} \mathrm{~S}$ donor, $\mathrm{NaHS}$, and endogenous $\mathrm{H}_{2} \mathrm{~S}$ within the PVN does not play a major role in the regulation of $\mathrm{BP}, \mathrm{HR}$, or LSNA in the normotensive state. In SHR rats, as in the WKY rats, we could not find any evidence suggesting $\mathrm{H}_{2} \mathrm{~S}$ in the PVN contributed to the regulation of the MAP, HR, and LSNA in the hypertensive state. Given the negative findings in the WKY rats, we did not pursue further investigations with HA and AOA in the PVN of SHR rats.

The RVLM is a key brain region involved in generating tonic sympathetic outflow (Guyenet, 2006). In the RVLM, microinjection of NaHS did not cause significant changes in MAP, HR, or LSNA compared to control. This was observed in WKY as well as SHR rats. The present results suggest that $\mathrm{H}_{2} \mathrm{~S}$ in the RVLM is not a key player in cardiovascular regulation in normotensive or hypertensive conditions. In order to observe the effects of endogenous $\mathrm{H}_{2} \mathrm{~S}$, two inhibitors of CBS were employed, HA and AOA (Johnston and Balcar, 1974; Vidrio and Medina, 2007). Since both inhibitors affect the association of CBS with its co-factor, pyridoxal-5' -phosphate (PLP), the effects could be attributable to inhibition of PLP-dependent enzymes other than CBS. However, neither inhibitor microinjected into the RVLM of WKY rats significantly affected MAP, HR, or LSNA. These results indicate that, although CBS is present in the RVLM, $\mathrm{H}_{2} \mathrm{~S}$ produced locally in the RVLM does not have a major influence on BP, HR, or LSNA. Since we did not see any evidence to suggest a role for endogenous $\mathrm{H}_{2} \mathrm{~S}$ in WKY rats nor for exogenous $\mathrm{H}_{2} \mathrm{~S}$ in WKY and SHR rats, we did not further investigate the effects of HA or AOA in the SHR rats.

In contrast to the present work, a study appearing immediately prior to submission of the present manuscript, reported that NaHS microinjected into the RVLM of anesthetized rats induced dosedependent, and relatively large, reductions in MAP, HR, and renal SNA (Guo et al., 2011). HA elicited the opposite cardiovascular effects (Guo et al., 2011). The reasons for the different responses compared to the present study are not known. In the present study the rats breathed spontaneously and a dorsal approach was used to functionally identify the pressor region of the RVLM. In the work by Guo et al., 2011, a ventral approach and visual identification of the RVLM was used, and the rats were ventilated. Stretching the chest wall during ventilation is known to enhance the excitatory drive arising from the RVLM and this can alter the responses to drugs administered into the RVLM (Cox and Brody, 1988). Functional identification of the pressor region of the RVLM was important in the present study to indicate (i) the correct placement of the microinjection and (ii) the RVLM was functional under the present experimental conditions and cardiovascular responses were clearly obtainable. It is noteworthy that similar doses were used in the present work and in the study by (Guo et al., 2011). It has also been reported that tachyphylaxis to repeated doses of NaHS may occur, at least in urogenital preparations (Patacchini et al., 2004). We do not believe this can account for the lack of responses in the present study since we have administered a single large dose of NaHS intracebroventricularly $(20 \mathrm{nmol})$ and found no significant effect on the cardiovascular variables. Additionally, $\mathrm{NaHS}$ is well known to induce vasorelaxation after sequential doses are administered (Al-Magableh and Hart, 2011; Hart, 2011). In two previous studies in which NaHS administration elicited significant changes in MAP and HR, the experiments were conducted 


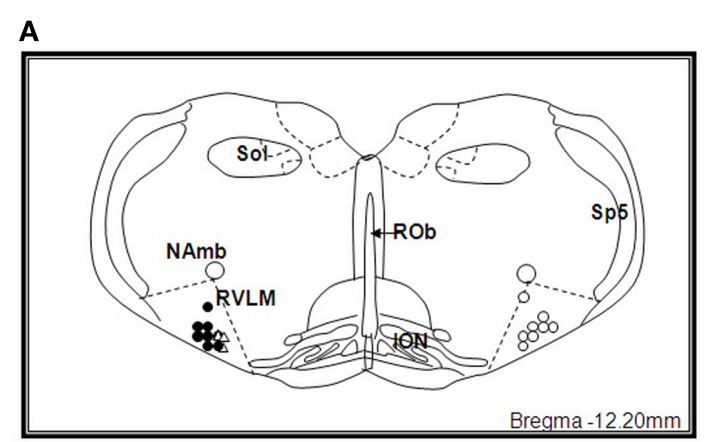

B

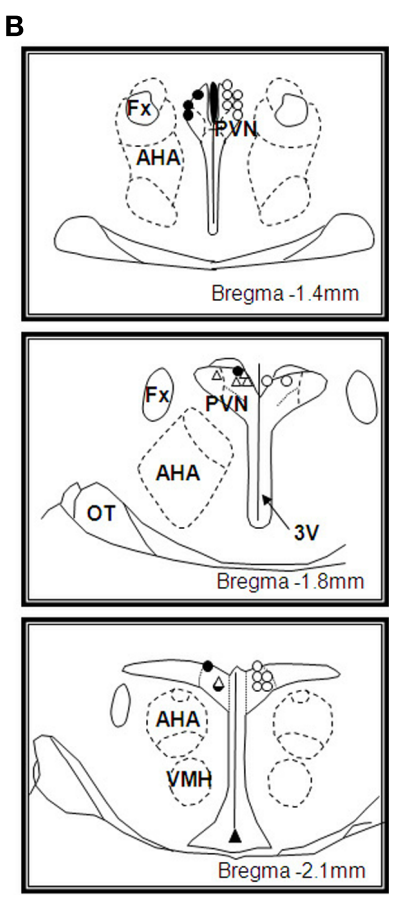

FIGURE 6 | (A) Schematic illustration showing the center of the microinjection sites within the rostral ventrolateral medulla (RVLM). Microinjections were made bilaterally but only unilateral sites are shown. Filled circles represent microinjection sites of $\mathrm{NaHS}$ and unfilled circles represent microinjection sites of amino-oxyacetate and hydroxylamine in WKY rats, unfilled triangles represent microinjection sites of NaHS in SHR rats. Approximate level caudal to bregma is indicated. Abbreviations: ROb, raphe obscurus; ION, inferior olivary nucleus; Sp5, spinal trigeminal tract; NAmb, nucleus ambiguous; Sol, solitary nucleus. (B) Schematic illustration showing the center of the microinjection sites within the hypothalamic paraventricular nucleus (PVN). Microinjections were made bilaterally but only unilateral sites are shown. Filled circles represent microinjection sites of $\mathrm{NaHS}$ and unfilled circles represent microinjection sites of amino-oxyacetate and hydroxylamine in WKY rats, unfilled triangles represent microinjection sites of NaHS in SHR rats. Approximate levels caudal to bregma are indicated. Abbreviations: Fx, fornix; AHA, anterior hypothalamic area; $3 \mathrm{~V}$, third ventricle; OT, optic tract; $\mathrm{VMH}$, ventromedial hypothalamus. in conscious rats. The changes in $\mathrm{BP}$ and $\mathrm{HR}$ reported were small (Dawe et al., 2008; Ufnal et al., 2008). The present work was conducted in the presence of anesthesia which may dampen $\mathrm{BP}$ and heart rate responses. Given the small magnitude of the responses to NaHS in the conscious rats, however, it is reasonable to question the physiological significance of $\mathrm{H}_{2} \mathrm{~S}$ in cardiovascular regulation.

Opening of $\mathrm{K}_{\mathrm{ATP}}$ channels is believed to contribute to the effects of $\mathrm{H}_{2} \mathrm{~S}$, including vasodilation and cardioprotection (Zhao et al., 2001; Bian et al., 2006). Opening $K_{\text {ATP }}$ channels could decrease cell firing as a result of hyperpolarization. Indeed, a reduced discharge rate in spontaneously firing units in the RVLM after administration of a $\mathrm{K}_{\mathrm{ATP}}$ channel opener, adenosine, has been reported but there was no effect on BP or HR (Chen and He, 1998). In the PVN, a recent in vitro study using hypothalamic slices showed that the spontaneous firing of PVN neurons with projections to the spinal cord was reduced by adenosine; an effect mediated by opening of $\mathrm{K}_{\text {ATP }}$ channels (Li et al., 2010). The present findings suggest that if $\mathrm{H}_{2} \mathrm{~S}$ opens $\mathrm{K}_{\mathrm{ATP}}$ channels in the RVLM or in the PVN, then $\mathrm{K}_{\mathrm{ATP}}$ channels in those brain regions have little influence in the regulation of MAP, HR, or LSNA.

Relatively high mRNA levels of the Kir6.2 subtype of the $\mathrm{K}_{\mathrm{ATP}}$ channel have been demonstrated in the PVN (Dunn-
Meynell et al., 1998). These channels may couple metabolic activity with neuronal excitability (Ashford et al., 1990), and are involved in sensing glucose and in regulating glucose metabolism (Zhang et al., 2004). Thus, although our studies suggest $\mathrm{H}_{2} \mathrm{~S}$ in the PVN has no major role in regulating $\mathrm{BP}, \mathrm{HR}$, and LSNA, $\mathrm{H}_{2} \mathrm{~S}$ in the PVN may perform other functions, which may involve metabolic regulation and these require further investigation.

\section{CONCLUSION}

We have demonstrated for the first time the presence of the enzyme CBS in two important cardiovascular regulatory regions, the RVLM and PVN. By contrast CSE was not observed in those brain regions. This is consistent with the current view that of those two enzymes, CBS is the main enzyme in the brain involved in the production of $\mathrm{H}_{2} \mathrm{~S}$. Our work also demonstrated there was no significant effect on BP, HR, and LSNA upon administration of the $\mathrm{H}_{2} \mathrm{~S}$ donor, NaHS, into the RVLM and PVN of WKY and SHR rats, or following inhibition of CBS in the RVLM and PVN in WKY rats. Thus, we suspect that $\mathrm{H}_{2} \mathrm{~S}$ in those regions is not playing a critical role in the regulation of $\mathrm{BP}, \mathrm{HR}$, and LSNA, at least, in the short term. 


\section{REFERENCES}

Abe, K., and Kimura, H. (1996). The possible role of hydrogen sulfide as an endogenous neuromodulator. $J$. Neurosci. 16, 1066-1071.

Al-Magableh, M. R., and Hart, J. L. (2011). Mechanism of vasorelaxation and role of endogenous hydrogen sulfide production in mouse aorta. Naunyn Schmiedebergs Arch. Pharmacol. 383, 403-413.

Ashford, M. L., Boden, P. R., and Treherne, J. M. (1990). Glucoseinduced excitation of hypothalamic neurones is mediated by ATPsensitive $\mathrm{K}+$ channels. Pflugers Arch. 415, 479-483.

Badoer, E. (2001). Hypothalamic paraventricular nucleus and cardiovascular regulation. Clin. Exp. Pharmacol. Physiol. 28, 95-99.

Badoer, E. (2010). Role of the hypothalamic PVN in the regulation of renal sympathetic nerve activity and blood flow during hyperthermia and in heart failure. Am. J. Physiol. Renal Physiol. 298, F839-F846.

Bian, J. S., Yong, Q. C., Pan, T. T., Feng, Z. N., Ali, M. Y., Zhou, S., and Moore, P. K. (2006). Role of hydrogen sulfide in the cardioprotection caused by ischemic preconditioning in the rat heart and cardiac myocytes. $J$. Pharmacol. Exp. Ther. 316, 670-678.

Calvert, J. W., Jha, S., Gundewar, S., Elrod, J. W., Ramachandran, A., Pattillo, C. B., Kevil, C. G., and Lefer, D. J. (2009). Hydrogen sulfide mediates cardioprotection through Nrf2 signaling. Circ. Res. 105, 365-374.

Cheang, W. S., Wong, W. T., Shen, B., Lau, C. W., Tian, X. Y., Tsang, S. Y., Yao, X., Chen, Z. Y., and Huang, Y. (2010). 4-Aminopyridinesensitive $\mathrm{K}(+)$ channels contributes to NaHS-induced membrane hyperpolarization and relaxation in the rat coronary artery. Vascul. Pharmacol. 53, 94-98.

Chen, F., Dworak, M., Wang, Y., Cham, J. L., and Badoer, E. (2008). Role of the hypothalamic PVN in the reflex reduction in mesenteric blood flow elicited by hyperthermia. Am. J. Physiol. Regul. Integr. Comp. Physiol. 295, R1874-R1881.

Chen, S., and He, R. R. (1998). Effect of intracarotid injection of adenosine on the activity of RVLM neurons in barodenervated rats. Sheng $\mathrm{Li}$ Xue Bao 50, 629-635.

Cox, B. F., and Brody, M. J. (1988). Tidal volume affects the response to inactivation of the rostral ventrolateral medulla. Hypertension 11, I186-I189.

Dawe, G. S., Han, S. P., Bian, J. S., and Moore, P. K. (2008). Hydrogen sulphide in the hypothalamus causes an ATP-sensitive $\mathrm{K}+$ channeldependent decrease in blood pressure in freely moving rats. Neuroscience 152, 169-177.

Deering, J., and Coote, J. H. (2000). Paraventricular neurones elicit a volume expansion-like change of activity in sympathetic nerves to the heart and kidney in the rabbit. Exp. Physiol. 85, 177-186.

Dunn-Meynell, A. A., Rawson, N. E., and Levin, B. E. (1998). Distribution and phenotype of neurons containing the ATP-sensitive $\mathrm{K}+$ channel in rat brain. Brain Res. 814, 41-54.

Gadalla, M. M., and Snyder, S. H. (2010). Hydrogen sulfide as a gasotransmitter. J. Neurochem. 113, 14-26.

Guo, Q., Jin, S., Wang, X. L., Wang, R., Xiao, L., He, R. R., and Wu, Y. M. (2011). Hydrogen sulfide in the rostral ventrolateral medulla inhibits sympathetic vasomotor tone through ATP-sensitive K+ channels. J. Pharmacol. Exp. Ther. 338, 458-465.

Guyenet, P. G. (2006). The sympathetic control of blood pressure. Nat. Rev. Neurosci. 7, 335-346.

Hart, J. L. (2011). Role of sulfurcontaining gaseous substances in the cardiovascular system. Front. Biosci. (Elite Ed) 3, 736-749.

Ishii, I., Akahoshi, N., Yu, X. N., Kobayashi, Y., Namekata, K., Komaki, G., and Kimura, H. (2004). Murine cystathionine gamma-lyase: complete cDNA and genomic sequences, promoter activity, tissue distribution and developmental expression. Biochem. J. 381, 113-123.

Johnston, G. A., and Balcar, V. J. (1974). Amino-oxyacetic acid: a relatively non-specific inhibitor of uptake of amino acids and amines by brain and spinal cord. J. Neurochem. 22, 609-610.
Kabil, O., and Banerjee, R. (2010). The redox biochemistry of hydrogen sulfide. J. Biol. Chem. 285 21903-21907.

Kantzides, A., Owens, N. C., De Matteo, R., and Badoer, E. (2005) Right atrial stretch activates neurons in autonomic brain regions that project to the rostral ventrolateral medulla in the rat. Neuroscience 133 , 775-786.

Kimura, H. (2002). Hydrogen sulfide as a neuromodulator. Mol. Neurobiol. 26, 13-19.

Kohl, R. L., and Quay, W. B. (1979). Cystathionine synthase in rat brain: regional and time-of-day differences and their metabolic implications. J. Neurosci. Res. 4, 189-196.

Lee, M., Schwab, C., Yu, S., Mcgeer E., and Mcgeer, P. L. (2009). Astrocytes produce the antiinflammatory and neuroprotective agent hydrogen sulfide. Neurobiol. Aging 30, 1523-1534.

Li, D. P., Chen, S. R., and Pan, H. L. (2010). Adenosine inhibits paraventricular pre-sympathetic neurons through ATP-dependent potassium channels. J. Neurochem. 113 530-542.

Mustafa, A. K., Gadalla, M. M., and Snyder, S. H. (2009). Signaling by gasotransmitters. Sci. Signal. 2, re2.

Patacchini, R., Santicioli, P., Giuliani, S. and Maggi, C. A. (2004). Hydrogen sulfide (H2S) stimulates capsaicinsensitive primary afferent neurons in the rat urinary bladder. $B r . J$. Pharmacol. 142, 31-34.

Robert, K., Vialard, F., Thiery, E., Toyama, K., Sinet, P. M., Janel, N. and London, J. (2003). Expression of the cystathionine beta synthase (CBS) gene during mouse development and immunolocalization in adult brain. J. Histochem. Cytochem. 51, 363-371.

Shafton, A. D., Ryan, A., and Badoer, E. (1998). Neurons in the hypothalamic paraventricular nucleus send collaterals to the spinal cord and to the rostral ventrolateral medulla in the rat. Brain Res. 801, 239-243.

Ufnal, M., Sikora, M., and Dudek, M. (2008). Exogenous hydrogen sulfide produces hemodynamic effects by triggering central neuroregulatory mechanisms.
Acta Neurobiol. Exp. (Wars) 68, 382-388.

Vidrio, H., and Medina, M. (2007). Hypotensive effect of hydroxylamine, an endogenous nitric oxide donor and SSAO inhibitor. J. Neural Transm. 114, 863-865.

Yang, G., Wu, L., Jiang, B., Yang, W., Qi, J., Cao, K., Meng, Q., Mustafa, A. K., Mu, W., Zhang, S., Snyder, S. H., and Wang, R. (2008). H2S as a physiologic vasorelaxant: hypertension in mice with deletion of cystathionine gamma-lyase. Science 322, 587-590.

Zhang, Y., Zhou, J., Corll, C., Porter, J. R., Martin, R. J., and Roane, D. S. (2004). Evidence for hypothalamic $\mathrm{K}+$ (ATP) channels in the modulation of glucose homeostasis. Eur. J. Pharmacol. 492, 71-79.

Zhao, W., and Wang, R. (2002). H(2)Sinduced vasorelaxation and underlying cellular and molecular mechanisms. Am. J. Physiol. Heart Circ. Physiol. 283, H474-H480.

Zhao, W., Zhang, J., Lu, Y., and Wang, R. (2001). The vasorelaxant effect of $\mathrm{H}(2) \mathrm{S}$ as a novel endogenous gaseous K(ATP) channel opener. EMBO J. 20, 6008-6016.

Conflict of Interest Statement: The authors declare that the research was conducted in the absence of any commercial or financial relationships that could be construed as a potential conflict of interest.

Received: 17 June 2011; accepted: 15 August 2011; published online: 01 September 2011.

Citation: Streeter E, Al-Magableh $M$, Hart JL and Badoer E (2011) Hydrogen sulfide in the RVLM and $P V N$ has no effect on cardiovascular regulation. Front. Physio. 2:55. doi: 10.3389/fphys.2011.00055

This article was submitted to Frontiers in Integrative Physiology, a specialty of Frontiers in Physiology.

Copyright () 2011 Streeter, Al-Magableh, Hart and Badoer. This is an open-access article subject to a non-exclusive license between the authors and Frontiers Media $S A$, which permits use, distribution and reproduction in other forums, provided the original authors and source are credited and other Frontiers conditions are complied with. 